\title{
The use of rheumatoid factors in clinical practice
}

\author{
Moira J Thomas, Adewale Adebajo, Helen M Chapel, Michael Webley
}

\begin{abstract}
Summary
Rheumatoid factor is a commonly requested test in the investigation of joint problems. Although the test has been found to be both sensitive and specific for rheumatoid arthritis in patients attending Rheumatology Clinics, when the test is applied to general hospital or community populations it performs poorly. Thus the setting in which rheumatoid factor tests are requested and awareness of the test's limitations are critical for appropriate use and interpretation of results. We studied 295 consecutive requests for testing at four centres. The majority were performed for the investigation of joint problems but only $6 \%$ of these were positive, suggesting unselective requesting. Half of the results were used to make diagnoses and influence management. However, significant numbers of clinicians felt that the test result had either excluded or confirmed a diagnosis of rheumatoid arthritis suggestging that results were being overinterpreted and that clinical decisions made on this basis may have been inappropriate. We conclude that in this setting rheumatoid factor testing is of limited value and generates misleading information.
\end{abstract}

Keywords: rheumatoid factor testing, audit

The rheumatoid factor (RF) has a long established place in rheumatological practice and is one of the most frequently requested investigations in patients with joint symptoms. A positive RF test has been reported to be present in up to $80 \%$ of patients with clinically and radiologically proven rheumatoid arthritis (RA) and it forms one of the criteria for the classification of this disease. ${ }^{1}$ However, a recent report on RFs in a large hospital population has cast doubt upon the clinical utility of the test in this setting, having found only $28 \%$ of RA patients to have a positive RF test. ${ }^{2}$ Since between $20 \%$ and $72 \%$ of patients with proven RA may be RF negative, serology is not diagnostic. Positive RFs also occur in healthy people, especially with increasing age or in association with infection. ${ }^{3}$ Such false-positive results have been reported to form a significant proportion of all RF requests ${ }^{2}$ and thus are a potential source of confusion. Both falsepositive and false-negative results could therefore lead to inappropriate diagnosis, treat- ment and referral. In this study we have looked at the pattern of $R F$ requests across the regiona health authority and the use made of the results.

\section{Methods}

The study was based in four Immunology Laboratories in one teaching hospital and three district general hospitals from the Oxfordshire Regional Health Authority. At the teaching hospital RF requests by non-rheumatologists were screened by first performing a C-reactive protein test. If this was normal, a RF test was not performed unless there was a particular clinical indication. At the three district general hospitals there was open access for all RF requests. All RF tests performed at the four centres over a four-week study period were identified and classified into three groups according to source of request - general practitioners, rheumatologists and other hospital clinicians and 25 consecutive requests from each requesting group at each centre were selected for further study. Requests for RF on joint aspirate were excluded. A questionnaire was sent to the clinician involved requesting the following information: reason for the test, pre- and post-test working diagnosis and the use made of the result for diagnosis, management and patient information.

RFs were performed initially by a latex agglutination test at three centres and then titred on a modified Rose Waaler Test (SCAT) in one centre and by Particle Agglutination (RAPA) Test at the other two centres. At the fourth centre all RFs were performed by RAPA testing. The RF was considered positive if the titre was $>1: 80$ by the specific SCAT or RAPA test. At two centres it was laboratory policy to $\frac{D}{D}$ perform a test for C-reactive protein on all samples tested for RF; at the other two centres $N$ this test was only performed on request.

\begin{tabular}{|l|l|}
\hline Causes of a positive RF & - rheumatoid arthritis \\
\hline - Siogren's syndrome \\
- type 2 cryoglobulinaemia \\
- infection \\
- healthy elderly \\
- normal population
\end{tabular}




\section{Results}

\section{SOURCE OF REQUESTS}

Overall, general practitioners accounted for the majority of requests $(52 \%)$; rheumatologists and other hospital clinicians accounted for $25 \%$ and $23 \%$, respectively. A total of 295 requests were selected as above for further study. The average age of this study population was 50 years (range $6-87$ years); the female to male ratio was $1.9: 1 ; 68 \%$ of the hospital requests came from general medical specialities, $16 \%$ from orthopaedics and $16 \%$ from assorted other specialties. There were no significant differences between the centres. Completed questionnaires were received from $82 \%$ of general practitioners, $76 \%$ of rheumatologists and $46 \%$ of other hospital clinicians.

\section{LABORATORY RESULTS}

Overall only $12 \%$ of RF tests were positive (ie, titres $>1: 80$ by SCAT or RAPA) and over half of these were in patients known to have seropositive RA. An erythrocyte sedimentation rate was performed on $67 \%$ and a C-reactive protein on $71 \%$ of all samples. Of note, in $55 \%$ of cases at one centre there was no laboratory assessment of the acute phase response (tables 1 and 2).

\section{REASON FOR RF REQUEST}

The main reason given for $R F$ requests was the investigation of joint symptoms or signs (table 1), although $13 \%$ were requested for the investigation of a wide range of other problems and $12 \%$ of requests for $\mathrm{RF}$ were made for the follow up of RA or seronegative arthritis $(64 \%$ of these patients had been retested within 12 months of the previous RF test).

\section{INFLUENCE OF RESULT ON THE WORKING DIAGNOSIS}

A total of $49 \%$ of respondents said that the RF result had 'changed or influenced their working diagnosis' with general practitioners more likely to be influenced by the result $(62 \%)$ than rheumatologists $(33 \%)$ (table 3$)$. Within this group, $50 \%$ had changed their working diagnosis compared with only $15 \%$ of those who felt that their diagnosis had not been influenced

Table 1 Reason for RF testing $(n=295)$

\begin{tabular}{lcc}
\hline Reason for $R F$ test & Number & Positive $R F$ \\
\hline Investigation of: & & \\
joint problem & 146 & 9 \\
other musculoskeletal problems & 8 & none \\
connective tissue disease & 4 & none \\
neurological disease & 2 & none \\
renal disease & 2 & none \\
liver disease & 3 & none \\
lung disease & 3 & none \\
systemic illness & 3 & none \\
Follow-up of: & & \\
known RA & 20 & 11 \\
seronegative arthritis & 3 & none \\
Inappropriate request & 4 & 1 \\
\hline
\end{tabular}

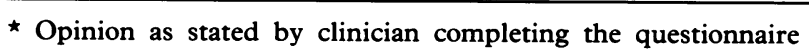

Table 2 Percentage of RF requests accompanied by measurement of C-reactive protein (CRP) and/or erythrocyte sedimentation rate (ESR)

\begin{tabular}{|c|c|c|c|c|}
\hline Centre & CRP alone & ESR alone & Neither & Both \\
\hline 1 & 16 & 39 & 55 & 9 \\
\hline $2^{\star}$ & 100 & 73 & 0 & 73 \\
\hline $3 \star$ & 99 & 73 & 1 & 73 \\
\hline 4 & 69 & 83 & 9 & 61 \\
\hline
\end{tabular}

* CRP measured routinely in conjunction with RF at these centres

by the result. A negative result was as likely as a positive result to influence diagnosis. Where the $R F$ result was said to have influenced the diagnosis, positive results were felt to confirm a diagnosis of RA in seven out of 10 positive results while negative results were stated to exclude a diagnosis of RA in 21 cases and to make this diagnosis less likely in a further 24 cases.

\section{INFLUENCE OF THE RESULT ON MANAGE-} MENT

Nearly half the respondents felt that the result had helped in management, again with a negative result having as much influence as a positive result (table 4). A total of $57 \%$ of patients were given specific information about the RF result. Negative results were used for 'reassurance' and to tell patients that RA had been excluded and positive tests were used to tell patients that RA had been confirmed.

\section{Discussion}

RFs were first discovered in 1940 in the serum of a patient with RA. ${ }^{4}$ Since then the RF has become one of the most frequently requested laboratory tests for the investigation of rheumatological problems. In recent years it has become clear that the clinical setting in which the RF is used has an important bearing upon the performance of the test. Whilst high values for the sensitivity and specificity of RFs have been demonstrated in patients attending rheumatological clinics, ${ }^{5}$ population studies of RF prevalence find only one person with RA for every seven positive RF tests. ${ }^{6}$ These factors clearly influence the interpretation of the $\mathrm{RF}$, therefore it is important to know how the test is being used in practice.

Our study examined the pattern of use of RF tests in one teaching and three district general hospitals. General practitioners were the main users of RF tests which were usually requested for the investigation of joint problems and the follow up of known RA. The small number of positive RFs in our study suggests over-use of the test, perhaps based on misconceptions about its sensitivity and specificity. Half of the requesters reported that the RF result (both positive and negative) influenced their diagnosis but the significant numbers of respondents stating that the RF result had either confirmed or excluded RA suggests that the RF results may well be over-interpreted. The RF is 
Table 3 Influence of RF result on diagnosis

\begin{tabular}{lcc}
\hline Reply & RF positive & RF negative \\
\hline RA confirmed & 7 & 2 \\
RA more likely & 1 & 0 \\
RA excluded & 0 & 21 \\
RA less likely & 0 & 24 \\
Other & & 2 \\
$\quad$ confirmed (unstated) working diagnosis & 0 & 2 \\
excluded connective tissue disease & 0 & 2 \\
seropositive RA excluded & 0 & 1 \\
helped rule-out systemic disease & 0 & 1 \\
excluded pathology & 0 & 32 \\
Unknown` & 2 & 87 \\
Total & 10 & \\
\hline
\end{tabular}

* Stated that RF influenced diagnosis but did not indicate how

Table 4 Effect of RF result on management

\begin{tabular}{llc}
\hline Effect of $R F$ result & $R F$ positive & $R F$ negative \\
\hline Result influenced therapy & & \\
start NSAIDs & 2 & 3 \\
avoid NSAIDs & 0 & 4 \\
start/consider DMRDs & 3 & 1 \\
avoid DMRDs & 1 & 4 \\
'change' in DMRDs & 1 & 0 \\
start steroids & 0 & 2 \\
avoid steroids & 0 & 1 \\
intra-articular steroids & 0 & 1 \\
non-drug therapy & 0 & 4 \\
Result influenced referral & & 12 \\
avoid hospital referral & 0 & 1 \\
refer to rheumatologist & 4 & 4 \\
refer to non rheumatologist & 0 & 5 \\
Result influenced further investigation & 0 & 15 \\
Result offered 'reassurance' & 0 & 7 \\
Result helped by clarifying diagnosis/prognosis & 0 & 20 \\
No change in management & 0 & 13 \\
Unknown & 2 & 88 \\
Total results which influenced management* & 10 & \\
\hline
\end{tabular}

NSAID: non-steroidal anti-inflammatory drug; DMRD: disease-modifying rheumatic drug. *Some results were felt to influence management in more than one way

included as part of the American Rheumatism Association 1987 revised criteria for the classification of $R^{1}$ but it is insufficient evidence on its own since positive RFs may be found in the normal population, in association with infection, with increasing age and with other disorders such as mixed essential cryoglobulinaemia and Sjogren's syndrome. ${ }^{3}$ In addition, between $22 \%$ and $72 \%$ of patients with definite RA are seronegative. ${ }^{2,5}$

Our study also shows that RF results influenced patient management. In many cases negative results simply reinforced the current management policy or provided 'reassurance' although this may have been unjustified in view of the frequency of seronegative RA. However, both positive and negative results were used to influence referral patterns and the choice of drug therapy especially the use of non-steroidal anti-inflammatory drugs and disease-modifying rheumatic drugs. $A$ positive $R F$ is associated with a poorer prognosis in $\mathrm{RA}^{7}$ and may justify the earlier use of disease-modifying rheumatic drugs provided other factors such as

\section{Learning points}

- a negative RF does not exclude rheumatoid arthritis $(20-72 \%$ of patients with RA are seronegative)

- high titre RF in association with RA indicates a worse prognosis, but levels do not follow disease activity closely

- a C-reactive protein and/or erythrocyte sedimentation rate may help identify the presence of an inflammatory arthritis; levels follow disease activity in RA

\begin{tabular}{|l|}
\hline Summary points \\
\hline - $74 \%$ of RFs were requested for the \\
investigation of joint problems \\
- $49 \%$ of results influenced diagnosis \\
- $48 \%$ of results influenced patient management \\
(eg, drug therapy, referral) \\
but \\
- low numbers of positive results suggest \\
unselective requesting \\
- use of RF result alone to exclude/confirm RA \\
suggests over-interpretation of results
\end{tabular}

the presence of a persistent acute phase response are also taken into account. However it is difficult to see how the RF result alone should alter the decision to use these drugs.

Active RA (and many other inflammatory arthritides) is accompanied by an acute phase response and indeed a persistently elevated C-reactive protein or erythrocyte sedimentation rate correlates with a poorer prognosis and earlier development of bony erosions. ${ }^{8}$ The former has some advantages over the latter in that it reflects the acute phase response alone and is unaffected by anaemia, fibrinogen, and viscosity, while its short half-life makes it more responsive to changes in disease activity. Although less specific for the acute phase response, the long half-life of the erythrocyte sedimentation rate may prove an advantage in some patients and may be raised in patients 을 with systemic lupus erythematosus where C- $\frac{D}{D}$ reactive protein is typically normal. Thus, measurement of either or both of these $N$ parameters adds useful information for the investigation of joint symptoms or for follow up. However, at one centre over half the clinicians requested neither test.

This study prompts us to suggest that guidelines for the use and interpretation of RFs would be helpful and such guidelines are currently in preparation. In general we would suggest that a C-reactive protein and/or an erythrocyte sedimentation rate is performed first to help identify whether an inflammatory arthritis is present and, where this is raised or there is clear clinical evidence of bilateral $\frac{\delta}{0}$ symmetrical synovitis, a RF may be helpful. We would also suggest that an anti-nuclear antibody be checked if there is clinical sus- 
picion of connective tissue disease. Once local consensus guidelines have been established it will be important to re-evaluate practice.

Our study confirms that, despite the widespread use of RFs, only a small number were actually positive, suggesting an unselective approach to requesting. Both positive and negative results were used to make diagnoses and change management but our study has highlighted some misconceptions about the interpretation of the test, suggesting that inappropriate diagnoses and decisions may have

1 Arnett FC, Edworthy SM, Bloch DA, et al. The American Rheumatism Association 1987. Revised criteria for the classification of rheumatoid arthritis. Arthritis Rheum 1988; 31: 315-24.

2 Shmerling RH, Delbanco TL. How useful is the rheumatoid factor? An analysis of sensitivity, specificity and predictive value. Arch Intern Med 1992; 152: 2417-20.

3 Shmerling RH, Delbanco TL. The rheumatoid factor: an analysis of clinical utility. Am $\mathcal{f}$ Med 1991; 91: 528-34.

4 Waaler $\mathrm{E}$. On the occurrence of a factor in human serum activating the specific agglutination of sheep blood corpuscles. Acta Pathol Microbiol 1940; 17: 172-88. been made. Recognition of the limitations of the RF test coupled with a much more selective approach to its request and greater use of measures of the acute phase response would reduce both costs and the amount of misleading information generated.

We are grateful to the following for their assistance in completing this study: Mr P Blackburn, Ms E Bolam, Ms F Legg, Mr K Walsh, Mrs J Wilkinson, Mrs J Wozniak and the clinicians who completed questionnaires.

5 Wolfe F, Cathey MA, Roberts RK. The latex test revisited. Arthritis Rheum 1991; 34: 951-60.

6 Valkenburg HA, Ball J, Burch TA, Bennett PH, Lawrence JS. Rheumatoid factors in a rural population. Ann Rheum Dis 1966; 26: 497-507.

7 McKenna F. Clinical and laboratory assessment of outcome in rheumatoid arthritis. $B r \mathcal{F}$ Rheumatol 1988; 27 (suppl 1): $12-20$

8 Amos RS, Constable TJ, Crockson RA, Crockson AP, McConkey R. Rheumatoid arthritis: relation of serum Creactive protein and erythrocyte sedimentation rates to radiographic changes. $B M \mathcal{F} 1977 ; 1$ : 195-7. 